\title{
Distribuição de mercúrio total em sedimentos da Plataforma Continental Amazônica - Brasil
}

\author{
Gilmar W. SIQUEIRA ${ }^{1}$, Fabio M. APRILE ${ }^{2, *}$ \\ RESUMO \\ Este estudo foi desenvolvido na Plataforma Continental do Amazonas (PCA) com o objetivo de determinar os níveis de $\mathrm{Hg}$ \\ total no sedimento de fundo, e assim contribuir como matriz ambiental indicadora para o plano geral de gerenciamento \\ costeiro na regiáo norte do Brasil. Foram amostrados 20 pontos entre maio e junho dos anos de 1999 a 2002, 2005 e 2007. \\ Os resultados indicam não haver evidências de atividades antrópicas relacionadas à contaminação de mercúrio na região. Os \\ teores encontrados variaram entre 0,047 e $0,166 \mathrm{mg} \mathrm{kg}^{-1}$ com média $0,085 \pm 0,026 \mathrm{mg} \mathrm{kg}^{-1}$, estando dentro do intervalo de \\ "background" referido para os rios amazônicos náo contaminados, que é de 0,05 a 0,28 $\mathrm{mg} \mathrm{kg}^{-1}$. As concentraçóes de $\mathrm{Hg}$ no \\ sedimento, especialmente no sedimento lamoso, mostraram uma estreita dependência com os teores de argila (material fino) e \\ matéria orgânica, corroborando mecanismos geoquímicos importantes na dinâmica do metal. A maior adsorçâo do mercúrio \\ pode estar associada ao aumento dos teores de compostos (óxidos e hidróxidos) de ferro, alumínio, manganês e os minerais \\ primários e secundários formadores das rochas da bacia de drenagem amazônica.
}

PALAVRAS-CHAVE: elemento metálico; matéria orgânica; sedimento de fundo; geoquímica; Amazônia.

\section{Distribution of total mercury in sediments from Amazon Continental Shelf - Brazil}

\section{ABSTRACT}

This study was developed at the Amazon Continental Shelf aims to determine the levels of total mercury in the sediment bottom, and them to contribute as environmental matrix for the general plan of coastal monitoring in the North Region of the Brazil. Twenty sampling sites between May and June from 1999 to 2002, 2005 and 2007 were analyzed. The results indicate there is not evidence of anthropic activities associated to the contamination by mercury in the region. The levels of total mercury founded range between 0.047 and $0.166 \mathrm{mg} \mathrm{kg}^{-1}$ with average of $0.085 \pm 0,026 \mathrm{mg} \mathrm{kg}^{-1}$. The results are inside of the background interval determined to not contaminated or not polluted Amazon Rivers, which the interval ranges from 0.05 to $0.28 \mathrm{mg} \mathrm{kg}^{-1}$. The mercury concentrations in the sediment bottom, especially in the mud sediment, had shown a narrow dependence with both the clay levels (fine material) and organic matter, corroborating important geochemical mechanisms in $\mathrm{Hg}$ dynamics. The largest adsorption of the mercury can be associated to the increase of the oxides and hydroxides compounds of iron, aluminum, manganese and others primary and secondary minerals from the rocks of the Amazon drainage basin.

KEYWORDS: metal; organic matter; bottom sediment; geochemical; Amazonian.

\footnotetext{
${ }^{1}$ Departamento de Química, Universidade Federal do Pará. Av. Augusto Corrêa n. 1, Campus Universitário do Guamá, 66075-100 Belém, PA, Brasil. Fone: (91)32017910. E-mail: gilmar@ufpa.br;

2 Laboratório de Biologia Ambiental, Universidade Federal do Oeste do Pará, Av. Marechal Rondon s/n Caranazal, 68040-070 Santarém, PA, Brasil. Fone: (93)30649066. E-mail: aprilefm@hotmail.com.

* Correspondence author.
} 


\section{INTRODUÇÃO}

A zona costeira brasileira compreende uma faixa terrestre de $20 \mathrm{~km}$ e uma faixa marítima de 6 milhas $(11,1 \mathrm{~km})$ de extensão a partir da linha de costa (Marins et al. 2004). Essa regiáo vem sendo ocupada desde os tempos coloniais e atualmente concentra cerca de $70 \%$ da população brasileira. A região da Plataforma Continental do Amazonas (PCA, 40 $\mathrm{N}-20^{\circ} \mathrm{S}$ ) se caracteriza por um ambiente dinâmico, devido à conjunção de vários fatores que interagem e produzem características oceanográficas únicas. A circulação da água nessa região representa uma interação do tipo estuarina, em um sistema fronteiro em direção ao mar a partir da desembocadura do rio Amazonas, com fortes rotativas de maré, especialmente nas direções N-NO e S-SE, e correntes de superfície resultantes da ação dos ventos na direção NO-SE (Siqueira 2003).

O rio Amazonas descarrega cerca de $18 \%$ de toda a descarga fluvial mundial nos oceanos, o que equivale em média $180.000 \mathrm{~m}^{3} \mathrm{~s}^{-1}$, com uma descarga sazonal mínima de $1,2 \times 10^{5} \mathrm{~m}^{3} \mathrm{~s}^{-1}$ em novembro e máxima de $2,5 \times 10^{5} \mathrm{~m}^{3}$ $\mathrm{s}^{-1}$ em maio (Goulding et al. 2003). Essa descarga fluvial transporta em conjunto uma imensa quantidade de material particulado em suspensáo (MPS), que segundo Meade et al. (1985), é da ordem de aproximadamente $1,2 \times 10^{9} \mathrm{t} \mathrm{ano}^{-1}$, que são continuamente depositadas ao longo da PCA. A descarga sólida do rio Amazonas gera importantes depósitos de sedimentos lamosos na Plataforma Continental Interna do Amazonas, o que gera um ambiente bastante distinto de outras áreas costeiras do planeta, com um imenso mosaico de sedimentos de fundo, variando do tipo arenoso ao lamoso rico em matéria orgânica. Estudos realizados por Pereira (2000); Siqueira e Braga (2000); Lima et al. $(2005,2006)$ e Siqueira et al. (2005) destacam a importância do MPS transportado pelos rios Amazonas e Pará, em direção a Plataforma Continental Interna, especialmente devido a sua capacidade de complexar aos sedimentos os metais, que à medida que passam pela zona estuarina em direção ao meio marinho, sofrem deposição sobre o sedimento de fundo oceânico. Dentre os metais distribuídos na PCA, este trabalho fez referência ao transporte e sedimentação do mercúrio $(\mathrm{Hg})$.

Reconhecem-se duas fontes principais de $\mathrm{Hg}$ para os ecossistemas aquáticos na Amazônia: as fontes naturais e antrópicas. O Hg é um elemento que está presente em condiçóes naturais na crosta terrestre, água, biota e atmosfera. (Lacerda et al. 2007). As fontes naturais são decorrentes de eventos tectônicos que promoveram a formação da bacia sedimentar em associação a derrames basálticos sobre o oceano, isso aproximadamente entre $2,5 \mathrm{Ba}$ no chamado ciclo Transamazônico, no Éon Proterozóico, e $450 \mathrm{Ma}$ na Era Mesozóica (Dall'Agnol e Rosa-Costa 2008). Juntos, esses eventos favoreceram o processo de mineralização de diversos metais como $\mathrm{Fe}, \mathrm{Mn}, \mathrm{Cu}, \mathrm{Au}, \mathrm{Ni}$ e $\mathrm{Hg}$. De acordo com Marins et al. (2004), processos naturais como a ressuspensão de partículas do solo pelos ventos, emanaçóes vulcânicas, queimadas de florestas e emanaçóes do solo e águas superficiais podem contribuir para as emissóes naturais de mercúrio para o ecossistema. Estudos demonstraram que as concentraçóes naturais de $\mathrm{Hg}$ na bacia do rio Negro excedem em muito qualquer possível fonte por atividade antrópica no local (Fadini e Jardim 2001; Rocha et al. 2003; do Valle et al. 2005). Nas áreas naturalmente contaminadas o $\mathrm{Hg}$ presente no solo é transportado para os corpos de água, onde pela atividade bacteriana é transformado em metilmercúrio, forma extremamente tóxica do elemento, que por sua vez tende a ficar biodisponível para diferentes reaçóes químicas, biológicas e fotoquímicas, podendo tornar-se bastante estável, bioacumulando na cadeia trófica e, por sua vez, contaminando toda a biota aquática, água e sedimento. Já a fonte antrópica está diretamente associada ao processo de produção de amalgama para recuperação do ouro de granulação fina, que por sua vez é queimado, geralmente ao ar livre, liberando o $\mathrm{Hg}$ para a atmosfera na forma de vapor.

As informaçóes sobre as quantidades de mercúrio utilizadas na atividade garimpeira e lançadas ao meio ambiente amazônico são extremamente contraditórias. A grande expansão da atividade mineradora iniciou-se na década de 1970, com o Plano de Integração Nacional, e teve seu auge entre as décadas de 1980 e 90, especialmente no sudoeste do Estado do Pará, próximo aos municípios de Itaituba, Jacareacanga e Eldorado dos Carajás, onde se encontra Serra Pelada. As bacias dos rios Madeira (RO) e Tapajós (PA) são responsáveis pela grande produçáo de ouro no Brasil, sendo esta ultima, na década de 1980, responsável por cerca de 50\% do ouro nacional garimpado (Santos et al. 2003). Para esse mesmo período, Lacerda e Pfeiffer (1992) estimaram que cerca de 1.500 a 3.000 ton de mercúrio foram lançadas na Amazônia Legal pela atividade garimpeira.

A análise dos teores de mercúrio no sedimento é uma das formas mais utilizadas de se avaliar o grau de contaminaçáo de um sistema aquático (Marins et al. 1998; Alonso et al. 2000). Devido a sua alta toxicidade e capacidade de sofrer biomagnificação em quase todas as cadeias tróficas, a exposição ambiental ao $\mathrm{Hg}$ via cadeia alimentar aumenta significativamente a exposição, principalmente em consumidores de nível trófico mais elevado, incluindo o homem (Agusa et al. 2007). Este trabalho objetivou determinar os níveis de $\mathrm{Hg}$ total no sedimento de fundo da Plataforma Continental do Amazonas, visando contribuir como matriz ambiental indicadora para o plano geral de gerenciamento costeiro na regiáo norte do Brasil. 


\section{MATERIAL E MÉTODOS}

As coletas foram iniciadas por ocasião do Programa Nacional de Avaliação do Potencial Sustentável de Recursos Vivos na Zona Econômica Exclusiva (REVIZEE-SCORE/ $\mathrm{NO}$ ), que ocorreu anualmente entre maio e junho, período de descarga mínima do rio Amazonas, dos anos de 1999 a 2002, e posteriormente continuadas para junho dos anos de 2005 e 2007. Foram selecionados 20 pontos na região entre as isóbatas de 19 a 100 m (Figura 1), e as coordenadas 47052'W-4¹9'N e $51^{\circ} 04^{\prime} W-2^{\circ} 16^{\prime} S$, dentro da Zona Econômica Exclusiva (ZEE), que se estende das 12 as 200 milhas náuticas marítimas. Em cada ponto foi amostrado cerca de $250 \mathrm{~g}$ de sedimentos de fundo $(0-5 \mathrm{~cm})$ com coletores tipo "Van Veen" e "Box Core", e subamostras foram acondicionadas em câmara fria a $-20{ }^{\circ} \mathrm{C}$. No campo ainda foram mensurados parâmetros físico-químicos como temperatura da água, $\mathrm{pH}$, condutividade elétrica e sólidos totais dissolvidos com auxílio de sondas portáteis modelo WTW e YSI, sendo que as amostras de sedimento foram retiradas após as mediçóes de água, para que se evitasse a ressuspensáo do material na mesma.

No laboratório de química da UFPA, as subamostras seguiram protocolo para preparaçáo, que se constituiu de secagem a $40 \pm 5^{\circ} \mathrm{C}$ por 48 horas, desagregação, pulverização, homogeneização, quarteamento e peneiramento (malha de $0,063 \mathrm{~mm}$ ou $230 \mathrm{mesh}$ ). Subamostras de sedimento seco foram utilizadas para análise granulométrica, através de um agitador mecânico com peneiramento fracionado. $\mathrm{O}$ teor

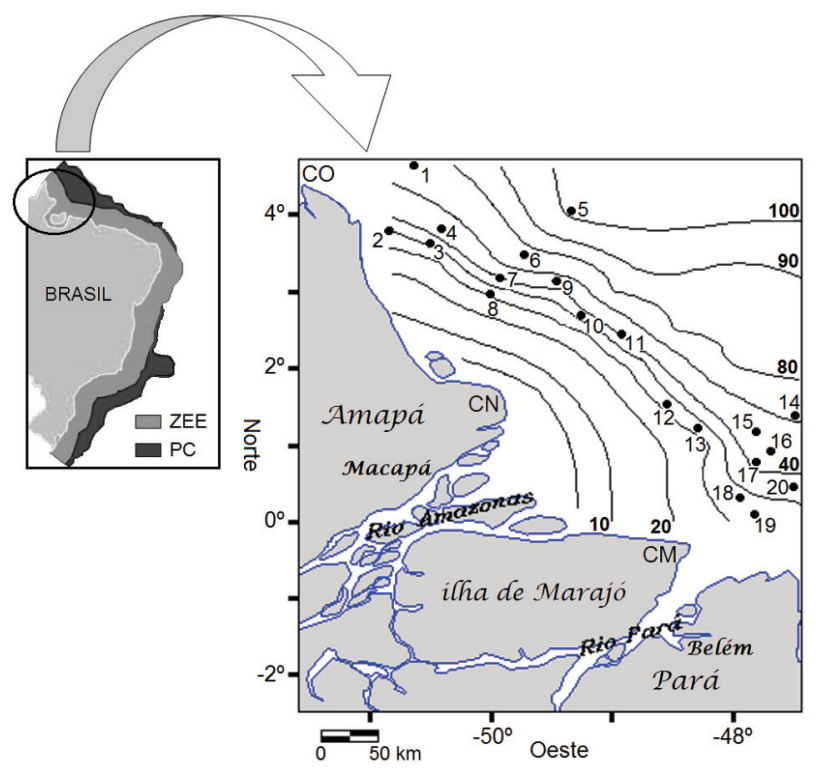

Figura 1 - Plataforma Continental Brasileira $\left(\mathrm{PC} \approx 960.000 \mathrm{~km}^{2}\right)$ e Zona Economicamente Exclusiva (ZEE $\approx 3.500 .000 \mathrm{~km}^{2}$ ), com destaque para Plataforma Continental do Amazonas (PCA), seus pontos de amostragem e suas respectivas isóbatas. (legenda: $\mathrm{CO}=\mathrm{Cabo}$ Orange; $\mathrm{CN}=\mathrm{Cabo}$ Norte; $\mathrm{CM}=$ Cabo Maguari). de matéria orgânica $(\mathrm{MO})$ foi determinado pelo método de extração ácida a quente, utilizando-se peróxido de hidrogênio $(30 \%)$ em chapa aquecedora a $100{ }^{\circ} \mathrm{C}$, pelo tempo necessário até extração completa da matéria orgânica, fato esse notado pela ausência de efervescência na amostra. Após a reação de extraçáo, o material foi secado e novamente pesado. A diferença entre o peso seco antes e depois da extração indicou a concentração de matéria orgânica [MO]. Já no laboratório da Lakerfild Geosol (MG), as amostras previamente preparadas seguiram os protocolos para abertura ou digestão e posterior leitura. Para a determinação de $\mathrm{Hg}$ total em sedimentos finos foram utilizados $1,000 \mathrm{~g}$ de amostras secas, colocadas em tubo de ensaio com $0,1000 \mathrm{~g} \mathrm{de} \mathrm{V}_{2} \mathrm{O}_{5}$. Posteriormente, adicionou-se aos tubos de ensaio $10 \mathrm{ml}$ de solução de água régia concentrada $\left(\mathrm{HCl}: \mathrm{HNO}_{3} ; 3: 1\right)$ para abertura das amostras. Os tubos foram então colocados em um bloco de alumínio para o aquecimento a $160{ }^{\circ} \mathrm{C}$ por 30 minutos em chapa aquecedora. Após os primeiros cinco minutos de digestão adicionou-se a mistura já aquecida $15 \mathrm{ml}$ de $\mathrm{H}_{2} \mathrm{SO}_{4}$. Ao término da digestáo, as amostras foram transferidas para balóes volumétricos de $100 \mathrm{ml} \mathrm{e}$ completado o volume com água deionizada (Milli-Q). Após, foram retiradas alíquotas e as leituras foram realizadas pelo método de geração de vapor frio em espectrometria de emissão óptica com fonte de plasma indutivamente acoplado (ICP), com limite de detecção de $0,01 \mathrm{ppm}\left(\mathrm{mg} \mathrm{kg}^{-1}\right)$. Os resultados foram certificados utilizando-se dois tipos diferentes de padrão para amostras de sedimento, o padrão internacional MAG-1 da Geostandards Newsletter e NIST 1646a. O folhelho médio foi utilizado como valor de referência para interpretação dos dados segundo Bowen (1979). Na preparação da curva padrão utilizou-se solução padrão Merck de $1000 \pm 1$ ppm, a qual foi diluída para determinação dos pontos da curva de massa de $\mathrm{Hg}$ (mg) e suas respectivas absorbâncias (Abs). Para determinação da concentração de $\mathrm{Hg}$ nas amostras, utilizou-se a equação 1:

$[\mathrm{Hg}]\left(\mathrm{mg}_{\mathrm{kg}} \mathrm{kg}^{-1}\right)=\frac{\text { massa } H g(\mathrm{mg}) \times \mathrm{F} . \mathrm{D} . \times \text { Vol total extrator }(\mathrm{ml})}{\text { aliquota }(\mathrm{ml}) \times \text { massa da amostra }(\mathrm{kg})}$

Onde: massa Hg é referente à massa de mercúrio (mg) relativo ao valor de absorbâncias da curva de calibração; F.D. é o fator de diluição, obtido a partir da quantidade total do extrato final $(\mathrm{ml})$ dividido pela alíquota $(\mathrm{ml})$ utilizada do extrato original; Vol é o volume total do extrator usado na digestão da amostra; alíquota é o volume utilizado para a leitura, e massa amostra total utilizada para digestão.

Análise de tendência central, histograma de distribuiçáo normal e análise descritiva múltipla foram aplicados aos resultados para uma avaliação estatística do comportamento do Hg total na PCA. 


\section{RESULTADOS}

Os resultados demonstram que as concentraçóes de $\mathrm{Hg}$ total encontradas nos sedimentos de influencia estuarina e costeira da PCA, para o período de estudo, não apresentaram grandes oscilaçóes em relação às médias pontual e geral, apresentando sempre baixos desvios-padrão. Os teores oscilaram entre 0,047 e $0,166 \mathrm{mg} \mathrm{kg}^{-1}$ com média geral de 0,085 $\pm 0,026 \mathrm{mg} \mathrm{kg}^{-1}$. Apesar da baixa variabilidade, entretanto, é possível destacar alguns pontos cujos valores médios foram ligeiramente mais elevados $\left(>0,100 \mathrm{mg} \mathrm{kg}^{-1}\right)$, sendo inclusive, notadas concentraçóes próximas do valor de referência de $0,180 \mathrm{mg} \mathrm{kg}^{-1}$ para folhelho médio citado por Bowen (1979), como foi o caso dos pontos 3, 9, 13 e 18 (Figura 2). Costa (2009), que estudou recentemente a distribuição de $\mathrm{Hg}$ total nos sedimentos ao longo do canal de maré da Gamboa do Cumbe, no rio Jaguaribe, já sob influência da zona costeira no Estado do Ceará, uma região ocupada com intensa atividade de carcinicultura, determinou teores de $\mathrm{Hg}$ que variaram aproximadamente entre 0,002 e $0,010 \mathrm{mg} \mathrm{kg}^{-1}$. Fazendo uma comparação entre os dados do autor e este trabalho nota-se que os teores de $\mathrm{Hg}$ encontrados na PCA chegaram à ordem de 25 vezes mais mercúrio no sedimento de fundo. A variabilidade espaço-temporal das concentraçóes de $\mathrm{Hg}$ total nos sedimentos de fundo da PCA é apresentada na Figura 3. Nota-se que ao longo dos seis anos de acompanhamento dos teores de mercúrio na região, os anos de 2001 e 2007 se destacaram, respectivamente, como sendo os anos de menor e maior concentração do elemento metálico no sedimento, não havendo, entretanto uma justificativa clara para tal comportamento. Os dados anuais confirmam a tendência anteriormente apresentada na Figura 2 (amplitude com valores médios), que demonstrou haver uma homogeneidade nos teores de mercúrio ao longo da Plataforma Continental. Os resultados demonstraram ainda haver uma tendência a maiores concentraçôes de mercúrio nos sedimentos lamosos, cuja composiçáo é predominantemente formada por argila mais material orgânico.

A Figura $4 \mathrm{~A}$ apresenta os isovalores de $\mathrm{Hg}$ total para a Plataforma Continental Amazônica, sendo que os resultados indicam uma homogeneidade que se estende do Cabo Orange, ao Norte da foz do rio Amazonas, descendo pelo Cabo Norte em direção ao Cabo Maguari, próximo à foz do rio Pará. Dentro dessa dispersão é possível localizar os pontos 3, 9 e 13 que ligados formariam uma linha diagonal em paralelo a linha da costa. A Figura 4B apresenta uma distribuição espacial dos grupos sedimentares encontrados na PCA. Nota-se a presença de três grupos: $1^{\circ}{ }_{-}$sedimentos arenosos, subdividido nas classes areia grossa (ponto 5) e média/fina (pontos 1 e 14); $2^{\circ}$ argilo-arenosos (ponto 17), e areno-argilosos (pontos 10 e 11); e $3^{\circ}$-sedimentos lamosos, formados por partículas finas, cuja composição é uma mistura de argila, areia, pequenas frações de silte e matéria orgânica (pontos 2 a 4, 6 a 9, 12, 13, 15, 16, 18, 19 e 20). Comparando-se as Figuras $4 \mathrm{~A}$ e $4 \mathrm{~B}$ confirma-se o que já foi anteriormente mencionado, que a maior concentração de $\mathrm{Hg}$ total foi determinada para a faixa compreendida pelos sedimentos lamosos, especialmente contendo argila e matéria orgânica. Esse fenômeno é conhecido por agregação ou formação coloidal, e é uma propriedade intrínseca tanto da argila quanto da $\mathrm{MO}$ em atrair elementos de carga positiva. A homogeneidade encontrada na regiáo pode significar ausência de fontes pontuais de mercúrio para a Plataforma Continental.

O significado dos níveis de contaminação de mercúrio no sedimento varia entre países e instituiçóes de proteção ambiental. A United States Environmental Protection Agency (US EPA 2003) considera que os sedimentos não poluídos devem apresentar teores de $\mathrm{Hg}$ total inferiores a $1,00 \mathrm{mg} \mathrm{kg}^{-1}$, enquanto que para o Ministério de Meio Ambiente de Ontário (Canadá) o valor de referência é $<0,3 \mathrm{mg} \mathrm{kg}^{-1}$, acima do qual o sedimento é considerado contaminado (Handy e Post 1985 apud Siqueira 2003). Já o Joint Group of Experts on the Scientific Aspects of Marine Pollution (GESAMP 1974), cita o teor de $0,1 \mathrm{mg} \mathrm{kg}^{-1}$ como sendo o limite máximo permitido

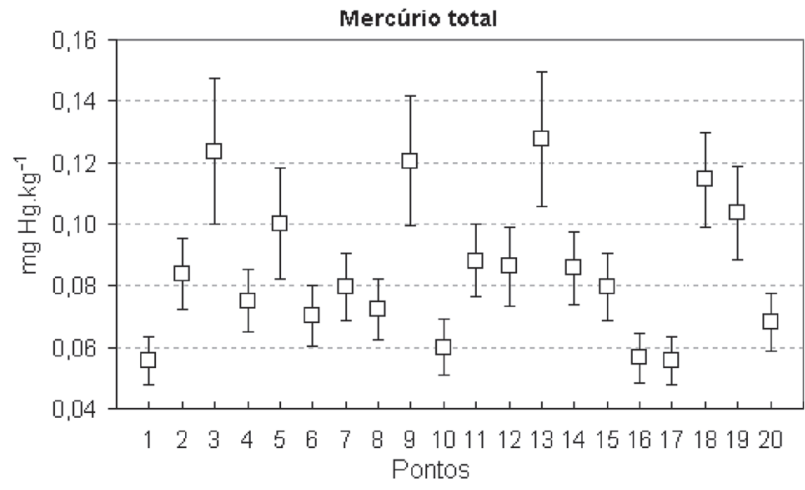

Figura 2 - Concentração média de $\mathrm{Hg}$ total nos sedimentos de fundo da Plataforma Continental Amazônica - PCA com seus respectivos desviospadrão.

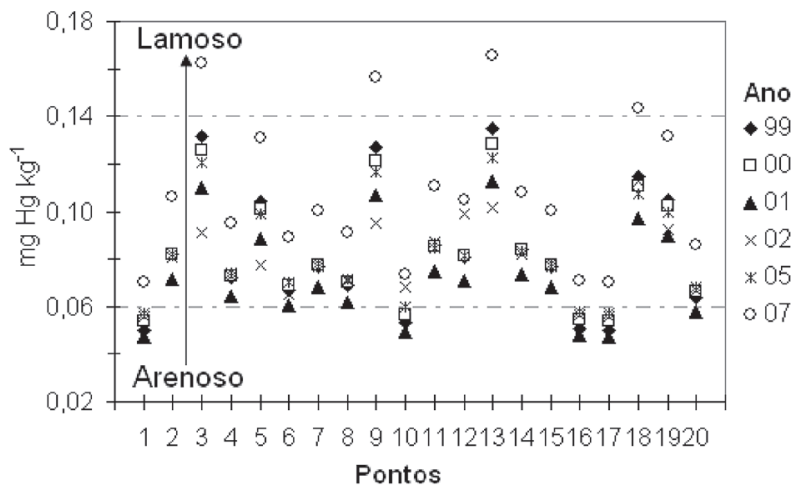

Figura 3 - Variabilidade espaço-temporal das concentrações de Hg total (mg $\mathrm{kg}^{-1}$ ) nos sedimentos de fundo da PCA. 
ou recomendado para $\mathrm{Hg}$ total em sedimentos de ambientes salobros e marinhos. Os teores encontrados para $\mathrm{Hg}$ total nos sedimentos da PCA estáo abaixo dos limites de referência das duas primeiras agências, para ambientes preservados, e muito próximos do valor recomendado pela GESAMP.

De acordo com Birch et al. (2000) apud Marins et al. (2004), vários estudos têm reportado que a média mundial
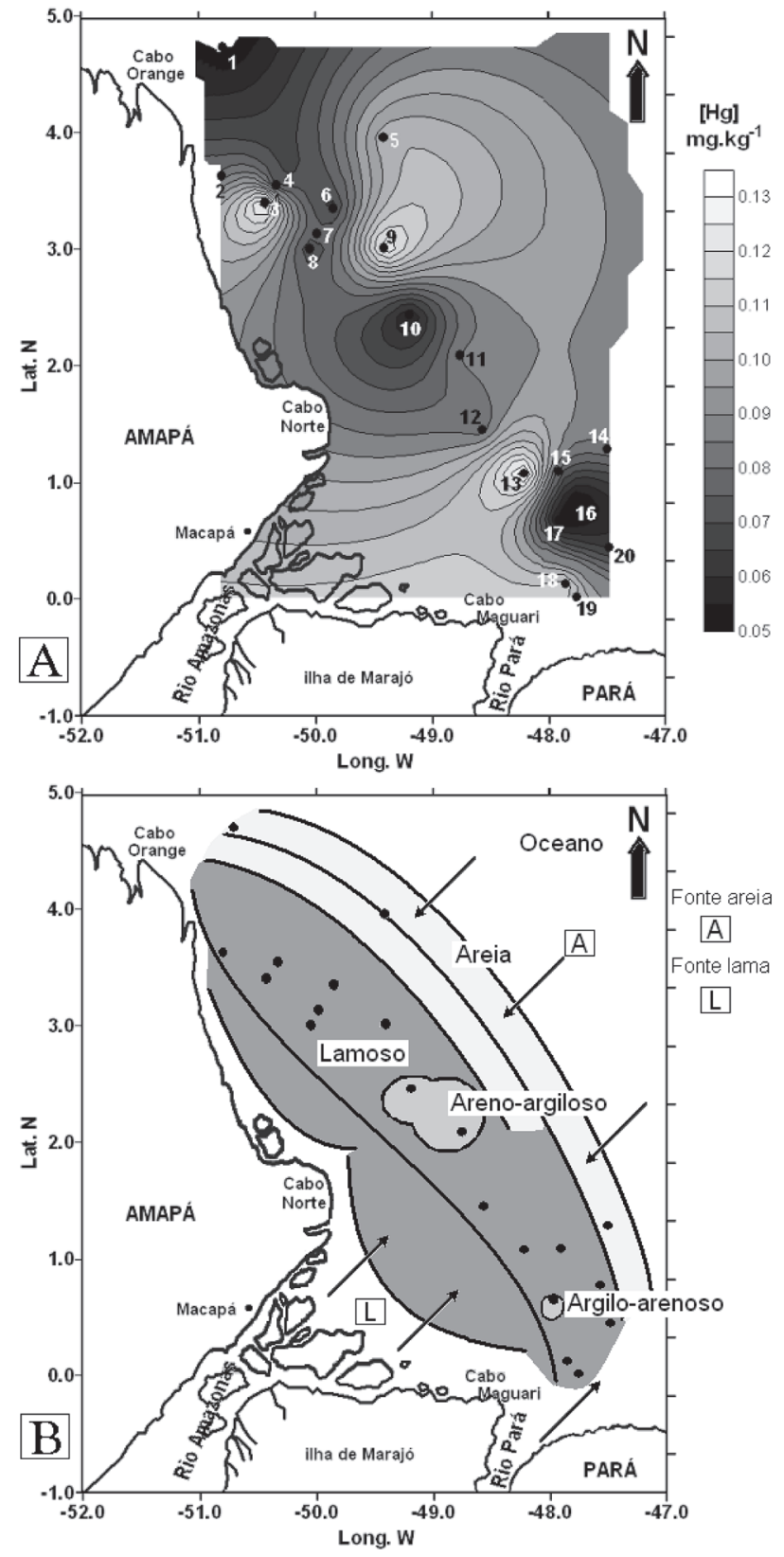

Figura 4 - A- Mapa de isovalores de $\mathrm{Hg}$ total na Plataforma Continental do Amazonas (PCA) para o período de amostragem entre 1999 e 2007; B- distribuição dos grupos sedimentares na área de estudo, indicando a tipologia do embasamento sedimentar e a influência oceânica e fluvial sobre o processo sedimentar.
("Clarke” geoquímico) e as concentraçóes médias de metais no folhelho são muito elevadas para representarem os níveis de "background" em diferentes bacias sedimentares. Diante deste cenário, tomou-se como base valores de "background" determinados para regiáo amazônica. Segundo Pfeiffer $e t$ al. (1989), os rios Amazônicos náo contaminados possuem teores de mercúrio associado ao sedimento de fundo aproximadamente inferiores a $0,20 \mathrm{mg} \mathrm{kg}^{-1}$. Dessa forma, observa-se que $100 \%$ das 120 amostras analisadas apresentaram níveis de $\mathrm{Hg}$ total abaixo do valor de "background" referido para os rios amazônicos não contaminados, cujo intervalo é de 0,05 a $0,28 \mathrm{mg} \mathrm{kg}^{-1}$. Comparativamente, os teores de $\mathrm{Hg}$ total encontrados na PCA são também muito mais baixos que os teores médios de $\mathrm{Hg}$ total $\left(0,240 \mathrm{mg} \mathrm{kg}^{-1}\right)$ determinados por Siqueira et al. (2005) para os sedimentos de fundo do Sistema Estuarino de Santos, onde há intensa atividade industrial.

Um histograma de distribuição normal de classes para as concentraçôes de $\mathrm{Hg}$ total foi elaborado e apresentado na Figura 5A. Com uma média de $0,085 \pm 0,026 \mathrm{mg} \mathrm{kg}^{-1}$, variância de 0,0006 e desvio do erro $<0,0021$ a classe predominante foi a do intervalo $0,0715-0,0855 \mathrm{mg} \mathrm{kg}^{-1}$ (ponto médio $0,0785 \mathrm{mg} \mathrm{kg}^{-1}$ ), que apresentou freqüência igual a 36 eventos. As maiores concentraçóes de $\mathrm{Hg}$ total, entretanto, náo representaram um grupo significativo na distribuiçấo
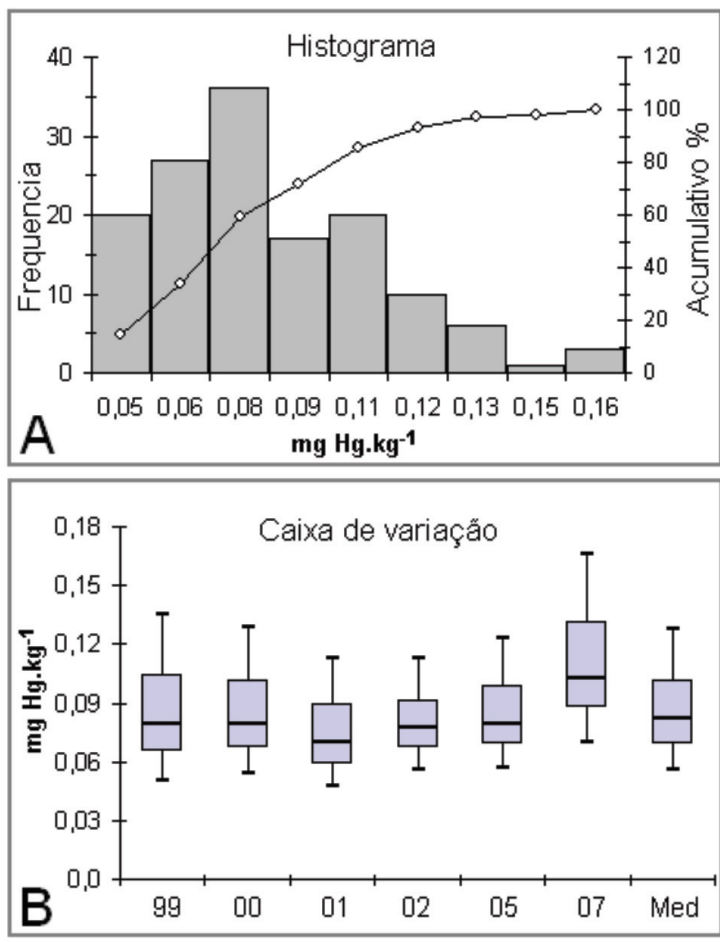

Figura 5 - A- Histograma de distribuição normal de classes para $0 \mathrm{Hg}$ total determinado na Plataforma Continental do Amazonas (PCA); B- distribuição em caixa das concentrações de $\mathrm{Hg}$ total na PCA em uma variação temporal com intervalo entre 1999 e 2007 
normal, somando-se juntas uma freqüência de 4 eventos para um intervalo entre $0,1415-0,1695 \mathrm{mg} \mathrm{kg}^{-1}$. Com base nos resultados já apurados e na análise de distribuiçáo normal, pode-se dizer que a homogeneidade encontrada na PCA está fortemente relacionada aos sedimentos lamosos, e que a concentração média determinada é representativa para o sistema. Fazendo ainda uma comparação dos teores de $\mathrm{Hg}$ total encontrados na Plataforma em uma distribuição temporal (Figura 5B), nota-se que a exceçáo do ano de 2007, cujas concentraçóes determinadas foram mais elevadas, todos os demais anos amostrados encontraram-se dentro da faixa de representatividade para o ambiente.

\section{DISCUSSÃO}

\section{Transporte e sedimentação metálica}

Dentro do contexto geoquímico, a deposição dos metais nos sedimentos de fundo dos ecossistemas aquáticos é facilitada na direção dos ambientes marinhos, visto os metais apresentarem baixa solubilidade em tais condiçôes. Além disso, o processo de transporte e deposição/sedimentação do material particulado em suspensão dependerá também de um conjunto de fatores físico-químicos, que incluem além da salinidade, o tempo de residência das águas, a baixa velocidade de fluxo de corrente, potencial redox, $\mathrm{pH}$, teor de matéria orgânica, tamanho das partículas, concentração de sulfetos e presença de bactéria sulfatoredutoras (Machado 2004; Cardoso et al. 2008; Reboreda et al. 2008). Segundo os autores ainda, ambientes aquáticos com maior tempo de residência ou que não apresentam competência de transporte possibilitam a deposição do material particulado em suspensão no sedimento, ficando a biodisponibilidade dos metais associados controlada aos fatores físico-químicos supracitados. Na regiáo de transição entre a foz dos rios Amazonas e Pará e o oceano, a zona de maré cria um ambiente de reduçáo da velocidade de corrente fluvial, atuando como uma forte barreira física ao fluxo dos rios, e em tais condiçóes, mudanças drásticas no $\mathrm{pH}$ e salinidade aceleram o processo de deposição sedimentar, que forma um imenso cordáo de sedimentos lamosos ricos em argilominerais facilitadamente associados aos elementos metálicos ao longo da costa Atlântica (como apresentado na Figura 4B). Nessa condição, processos físicos e químicos como: absorção, troca catiônica, precipitação, co-precipitação, complexação/ floculaçáo retêm, no sedimento de fundo, os metais trocáveis e os associados a diversos substratos geoquímicos.

Para a compreensão dos processos biogeoquímicos, que controlam o comportamento e a disponibilidade do $\mathrm{Hg}$ nos ambientes estuarino e oceânico, é necessário conhecer o padrão de variação das características físicas, químicas e físicoquímicas dos ecossistemas associados, o ciclo hidrológico, a sazonalidade na bacia, o efeito do pulso de inundação, as características geomorfológicas, uso e ocupação dos solos marginais, além de procurar determinar as diferentes espécies químicas de $\mathrm{Hg}$ no ambiente. As principais vias de acesso do $\mathrm{Hg}$ para as regiôes estuarina são a deposição atmosférica, liberação direta por efluentes e lixiviação de solos (Bisinot e Jardim 2004; Miretzsk et al. 2005). Destas, os efluentes da atividade mineradora e a lixiviaçáo dos solos merecem destaque na regiáo Amazônica, onde a mineraçáo está concentrada especialmente nos Estados de Rondônia e Pará, através da exploração de leito de rio, cavas e barrancos, que acabam por liberar o metal associado à grande carga de material sedimentar para a coluna de água. Já a lixiviação dos solos é bastante intensificada em toda a planície de inundação, especialmente nas bacias de águas brancas ou barrentas, onde o fenômeno de 'terras caídas' contribui significativamente para erosão e transporte de argilominerais e matéria orgânica. As áreas inundadas apresentam grande sensibilidade a poluentes como o Hg, náo apenas concentrando-o no sistema, mas também facilitando reaçôes biogeoquímicas que podem modificar sua estrutura química, mobilização e biodisponibilidade, cujo resultado é o aumento do potencial de toxicidade (Lacerda e Fitzgerald 2001; Oliveira et al. 2007).

A forma particulada é a forma dominante de $\mathrm{Hg}$ no processo de lixiviaçáo dos solos, sendo esta influenciada pela deposição oriunda de atividades antrópicas (Lacerda e Gonçalves 2001; Lacerda et al. 2007). Já o Hg dissolvido, não diferentemente de outros elementos metálicos, é fortemente adsorvido nos sedimentos e MPS, incluindo a matéria orgânica, e principalmente nos óxidos e hidróxidos de Fe e Mn. O tamanho do gráo é outro fator preponderante na disponibilidade do $\mathrm{Hg}$ para a coluna de água, sendo que uma vez depositado em sedimentos estuarinos ricos em matéria orgânica o $\mathrm{Hg}$ reduz os seus efeitos deletérios, podendo haver disponibilização para a coluna de água por meio da atividade biológica, como a bioturbação (Cardoso et al. 2008), que pode formar estruturas metiladas biodisponíveis. $\mathrm{O}$ processo laterítico na Amazônia produz sedimentos ricos em óxidos hidratados associados ao material argiloso, especialmente de $\mathrm{Fe}$, e na Plataforma Continental Interna assim como no delta do Amazonas, tem-se notado uma intensa laterizaçáo. $\mathrm{O}$ rio Amazonas transporta cerca de $82 \%$ do Fe em suspensão, carreando numerosos metais-traço (Melfi e Carvalho 1983). Alguns desses estudos têm destacado a importância de argilominerais e hidróxidos e óxidos hidratados de Fe e Mn no transporte de metais nos sedimentos da PCA (Pereira 2000; Siqueira e Braga 2000; Siqueira 2003; Siqueira et al. 2005; Lima et al. 2005), e outros o papel do MPS e matéria orgânica associada no transporte e complexação dos metais (Machado 2004; Paraquetti et al. 2004; Agusa et al. 2007; Cardoso et al. 2008; Reboreda et al. 2008).

A identificação da tipologia sedimentar da foz do rio Amazonas, bem como da Plataforma Continental, permitiu confirmar a evidencia de que os metais tem especial associação 
com argilominerais, e que essa associação é intensificada na presença de matéria orgânica. Associado a esse fato, as altas concentraçốes naturais de $\mathrm{Hg}$ nos solos das bacias dos rios Tapajós e Negro e a sua liberação pelo processo de podzolização (Fadini e Jardim 2001; Rocha et al. 2003; do Valle et al. 2005, 2006; Grimaldi et al. 2008), permitem a distribuição espontânea do metal para os ecossistemas aquáticos. Dessa maneira, justifica-se a presença de altas concentraçóes de $\mathrm{Hg}$ em pontos distantes de áreas de garimpo e, portanto, explica porque os teores de $\mathrm{Hg}$ total na PCA estáo particularmente mais altos no setor interno da área de estudo, estendendo-se desde o Cabo Orange até o Cabo Maguari, exatamente onde ocorre o processo de sedimentação fluvial oriunda dos rios Amazonas e Pará (Figura 4B).

\section{Geoquímica da Plataforma}

Para Queiroz (1995) apud Siqueira (2003), em pH básico o mercúrio tem uma maior afinidade pela fração mineral, desfavorecendo a formação do metil-Hg. Na PCA o $\mathrm{pH}$ variou entre 7,7 e 8,5 (média $8,1 \pm 0,2$ ) nas camadas de águas superficiais, e entre 8,0 e 8,2 (média $8,1 \pm 0,1$ ) nas camadas mais profundas, mantendo-se sempre ligeiramente básico. Dessa forma, sugere-se que os conteúdos de $\mathrm{Hg}$ total determinados estáo associados aos minerais primários e secundários existentes nessa região. Essa hipótese é embasada em alguns dos primeiros estudos mineralógicos realizados na PCA, como o de Pomerancblum e Costa (1972), que identificaram várias províncias de minerais pesados na regiáo. Esses autores encontraram como assembléias mineralógicas típicas da região o hiperstênico, hornblenda, biotita, enstatita, sillimanita e diopsídio. Barreto et al. (1975) apud Siqueira (2003) identificaram suítes de minerais pesados associados às areias arcoseanas (hornblenda, enstatita, hiperstênio e sllimanita) e as areias quartzosas (estaurolia, turmalina, zircão e cianita) próximo à foz do rio Amazonas. Ivo e Figueiredo Junior (1996) encontraram alguns minerais representativos para essa Plataforma, tais como: magnetita, ilmenita, óxido hidratado, hornblenda, hiperstênio, augita, epídoto, andalusita, granada, biotita, turmalina, estaurolita e zircáo. Muitos desses minerais, que estão na forma de argilominerais na PCA, especialmente os óxidos de ferro magnetita $\left[\mathrm{Fe}_{3} \mathrm{O}_{4}\right]$ e ilmenita $\left[\mathrm{FeTiO}_{3}\right]$, os silicatos andalusita $\left[\mathrm{Al}^{5} / \mathrm{Al}^{6} \mathrm{OSiO}_{4}\right]$ e granada $[(\mathrm{Fe}, \mathrm{Mg})$ $\left.\mathrm{Al}_{2}\left(\mathrm{SiO}_{4}\right)_{3}\right]$ e a mica biotita $\left[\mathrm{K}(\mathrm{Mg}, \mathrm{Fe})_{3}\left(\mathrm{Al}, \mathrm{Si}_{3} \mathrm{O}_{10}\right)(\mathrm{OH})_{2}\right]$ tem um importante papel na ligação e consequente transporte dos metais como mercúrio. Na regiáo da foz dos rios Amazonas e Pará, tais argilominerais são abundantes e transportados dos solos marginais (terra-firme) pelos intensos processos de erosão hídrica e lixiviação.

A afinidade dos metais, especialmente neste caso a do $\mathrm{Hg}$, pela fração mineral também leva em consideração o tamanho da partícula. Do Valle et al. (2005) encontraram diferenças nas concentraçóes de $\mathrm{Hg}$ quando comparados às diferentes fraçôes granulométricas dos Latossolos e Podzóis. Para os autores, há uma forte correlaçáo entre o metal, os teores de carbono orgânico e a granulometria das partículas, especialmente nos podzóis. $\mathrm{O}$ acúmulo de matéria orgânica associada aos ácidos húmico e fúlvico em rios de águas pretas é crucial para explicar o comportamento, concentração e transporte do Hg nessas águas (migração vertical e lateral e complexaçáo de organometálicos), da mesma maneira que o acúmulo de argilominerais explica tais fenômenos nos rios de águas brancas.

Lima et al. (2005) estudando a composição mineralógica dos sedimentos de fundo na PCA, observou picos de 7 e 3,6 $\AA ̊$, que são característicos de caulinita $\left[\mathrm{Al}_{2} \mathrm{Si}_{2} \mathrm{O}_{5}(\mathrm{OH})_{4}\right] ; 15,3$ $\AA \AA$, que permite identificar a montmorillonita (esmectita) $\left[\mathrm{Al}_{2} \mathrm{Si}_{4} \mathrm{O}_{10} \cdot \mathrm{nH}_{2} \mathrm{O}\right]$; picos de 10,5 e $3,3 \AA$, que indicam ocorrência de ilita $\left[\mathrm{KAl}_{2}(\mathrm{SiAl})_{4} \mathrm{O}_{10}(\mathrm{OH})_{2} \cdot \mathrm{nH}_{2} \mathrm{O}\right]$ e gibbsita $\left[\mathrm{Al}_{2} \mathrm{O}_{3} \cdot 3 \mathrm{H}_{2} \mathrm{O}\right]$, todos minerais com razoável poder de adsorçấo. Para Jonasson e Boyle (1979) apud Siqueira (2003), em decorrência da coexistência de depósitos de cinabar, metacinabar ou outros minerais a base de mercúrio, o conteúdo de $\mathrm{Hg}$ total nos minerais pode variar com a composição (em porcentagem) da seguinte forma: tetraedrita $\left[(\mathrm{Cu}, \mathrm{Fe})_{12} \mathrm{Sb}_{4} \mathrm{~S}_{13}\right]$ comumente em ligas de cobre, chumbo e bismuto - 17,6 a $21 \%$; minerais de cobre cinzas $(\mathrm{Cu}, \mathrm{As}$ e $\mathrm{Sb})-14 \%$; aragonita $\left(\mathrm{CaCO}_{3}\right)-3,5 \%$; realgar $[\mathrm{AsS}]-2,2 \%$; pirolusita $\left[\mathrm{MnO}_{2}\right]$ - 2\%; pirita $[\mathrm{FeS}]-2 \%$ e galena $[\mathrm{PbS}]-0,02 \%$, ambos sulfetos cúbicos; estibinita $\left[\mathrm{Sb}_{2} \mathrm{~S}_{3}\right]-1,3 \%$; esfarelita $[\mathrm{ZnS}]$ em depósitos sedimentares e de filiação vulcanogênica $-1 \%$; barita $\left(\mathrm{BaSO}_{4}\right)-0,5 \%$; óxidos hidratados de ferro $\left[\mathrm{Fe}_{2} \mathrm{O}_{3}\right.$. $\left.\mathrm{nH}_{2} \mathrm{O}\right)-0,2 \%$; calcita $\left(\mathrm{CaCO}_{3}\right)-0,03 \%$; wurtizite $[\mathrm{ZnS}]-$ $0,03 \%$; cerussita $\left(\mathrm{PbCO}_{3}\right)$ decorrente da oxidação supérgena da galena; fluorita $\left[\mathrm{CaF}_{2}\right]-0,01 \%$; siderita $\left(\mathrm{FeCO}_{3}\right)-0,01 \%$. Em oposição ao padrấo de argilominerais fornecido pelos sistemas fluviais amazônicos, encontram-se os sedimentos quartzíticos $\left[\mathrm{SiO}_{2}\right]$ oriundos do oceano, contendo pouca matéria orgânica, e que tem baixa capacidade de adsorçáo metálica. O que confirma o padrão de concentração mercurial encontrado no eixo orientado de descarte fluviomarinho concentrado na foz dos rios Amazonas e Pará.

\section{CONCLUSÕES}

Os resultados indicam uma homogeneidade nos níveis de $\mathrm{Hg}$ total nos sedimentos de fundo da Plataforma Continental do Amazonas, que se estende do Cabo Orange, ao Norte da foz do rio Amazonas, em direção ao Cabo Maguari, próximo à foz do rio Pará. Foi observado náo haver existência de evidências de atividades antrópicas reconhecidamente de fontes de mercúrio para a região da Plataforma estudada. Os teores encontrados para $\mathrm{Hg}$ total nos sedimentos da PCA estão abaixo dos limites de referência de importantes agencias ambientais internacionais, citando United States 
Environmental Protection Agency (US EPA 2003) e Ministério de Meio Ambiente de Ontário. A maior adsorção do mercúrio possivelmente pode estar associada ao aumento dos teores de compostos (óxidos e hidróxidos) de ferro, alumínio, manganês e os minerais primários e secundários formadores das rochas da bacia amazônica. Além disso, notadamente há uma relação direta entre a adsorção do metal nos sedimentos com maior concentração de argila e matéria orgânica, definidos como sedimentos lamosos. Apesar dos resultados positivos, torna-se necessário a continuidade dos estudos para o bom andamento do programa de gerenciamento costeiro da região.

\section{BIBLIOGRAFIA CITADA}

Agusa, T.; Kunito, T.; Sudaryanto, A.; Monirith, I.; Kan-Atireklap, S; Iwata, H; Ismail, A.; Sanguansin, J.; Muchtar, M; Tana, T.S.; Tanabe, S. 2007. Exposure assessment for trace elements from consumption of marine fish in Southeast Asia. Environmental Pollution, 145: 766-777.

Alonso, D.; Pineda, P.; Olivero, J.; González, H.; Campos, N. 2000. Mercury levels in muscle of two fish species and sediments from the Cartagena Bay and the Ciénaga Grande de Santa Marta, Colombia. Environmental Pollution, 109(1): 157-163.

Bisinot, M.C.; Jardim, W.F. 2004. Behavior of methylmercury in the envorinment. Quimica Nova, 27(4): 593-600.

Bowen, H.J.M. 1979. Environmental chemistry of the elements. London Academic Press, London. 273p.

Cardoso, P.G.; Lilllebo, A.I.; Lopes, C.B.; Pereira, E.; Duarte, A.C.; Pardal, M.A. 2008. Influence of bioturbation by Hediste diversicolor on mercury fluxes from estuarine sedmints: A Mesocosms laboratory experiment. Marine Pollution Bulletin, 56: 315-334.

Costa, B.G.B. 2009. Distribuição de mercúrio (Hg) em sedimento, água e biota da regiāo de influencia dos efluentes da carcinicultura, na Gamboa do Cumbe - rio Jaguaribe - Ceará - Brasil. Master's Thesis, Ciências Marinhas Tropicais, Universidade Federal do Ceará. 60p.

Dall'Agnol, R.; Rosa-Costa, L.T. 2008. A formação do continente Amazônico. Scientific America Brasil, Coleção Amazônia. Ed. Duetto, São Paulo, SP. p. 6-13.

Do Valle, C.M.; Santana, G.P.; Augusti, R.; Egreja Filho, F.B.; Windmöller, C.C. 2005. Speciation and quantification of mercury in Oxisol, Ultisol, and Spodosol from Amazon (Manaus, Brazil). Chemosphere, 58(6): 779-92.

Do Valle, C.M.; Santana, G.P.; Windmöller, C.C. 2006. Mercury conversion processes in Amazon soils evaluated by thermodesorption analysis. Chemosphere, 65(11): 1966-1975.

Fadini, P.S.; Jardim, W.F. 2001. Is the Negro River Basin (Amazon) impacted by naturally occurring mercury? The Science of the Total Environment, 275(1-3): 71-82.

GESAMP. 1974. Aspects of marine pollution. Report of sixth session 22-28 m1rc, Geneva. 43p.
Goulding, M.; Barthem, R.; Ferreira, E. 2003. The Smithsonian atlas of the Amazon. Princeton Editorial Associate, Inc. Hong Kong. 253p.

Grimaldi, C.; Grimaldi, M.; Guedron, S. 2008. Mercury distribution in tropical soil profiles related to origin of mercury and soil processes. The Science of the Total Environment, 401: 121-129.

Ivo, J.M.F.; Figueiredo Junior, A.G. 1996. Concentraçóes de minerais pesados associados à morfologia da Plataforma Continental do Amazonas, p. 440-442. In: Anais do XXXIX Congresso Brasileiro de Geologia.

Lacerda, L.D.; Santos, A.F.; Marins, R.V. 2007. Emissão de Mercúrio para a atmosfera pela queima de gás natural no Brasil. Química Nova, 30(2): 366 - 369 .

Lacerda, L.D.; Pfeiffer, W.C. 1992. Mercury from gold mining in the amazon environmental: an overview. Quimica Nova, 15(2): 155-160.

Lacerda, L.D.; Gonçalves, G.O. 2001. Mercury distribution and speciation in waters of the coastal lagoons of Rio de Janeiro, SE Brazil. Marine Chemistry, 76: 47-58.

Lacerda, L.D.; Fitzgerald, W.F. 2001. Biogeochemistry of mercury in wetlands. Wetlands Ecology and Management, 9: 291-293.

Lima, E.A.R.; Lima, W.N.; Siqueira, G.W.; Siqueira, N.V.M. 2005. Influência da mineralogia na composição química de metais nos sedimentos pelíticos superficiais da Plataforma Continental do Amazonas. Geochimica Brasiliensis, 19(2): 103-117.

Lima, E.A.R.; Siqueira, G.W.; Lima, W.N. 2006. Utilização dos critérios de avaliação ambiental de metais pesados nos sedimentos de fundo da plataforma continental do Amazonas. Boletim Museu Paraense Emílio Goeldi, 1(1): 105-114.

Machado, W.T.V. 2004. Fatores de influência sobre a capacidade de retenção de metais pesados por sedimentos de manguezal. Master's Thesis, Geociências, Universidade Federal Fluminense, Niterói, RJ. p.141.

Marins, R.V.; Filho, F.P.; Maia, S.R.R.; Lacerda, L.D.; Marques, W.S. 2004. Distribuição de mercúrio total como indicador de poluição urbana e industrial na costa brasileira. Quimica Nova, 27(5): 763-770.

Marins, R.V.; Lacerda, L.D.; Paraquetti, H.H.M.; Paiva, E.C. de; Villas Boas, R.C. 1998. Geochemistry of mercury in sediments of a sub-tropical coastal lagoon, Sepetiba Bay, Southeastern Brazil. Bulletin of Environmental Contamination and Toxicology, 61(1): 57-64.

Meade, R.H.; Dunne, T.; Richey, U.M.S.; Salati, E. 1985. Storage and remobilization of suspended sediment in the lower Amazon river of Brazil. Science, 228: 488-490.

Melfi, A.J.; Carvalho, A. 1983. Soil, sediment, major formation of the continental biosphere. Anais da Academia Brasileira de Ciências, 66(1): 195-203.

Miretzsk, M.C.; Bisinot, M.C.; Jardin, W.F. 2005. Factors affecting $\mathrm{Hg}$ adsorption in soils from the Rio Negro Basin (Amazon). Quimica Nova, 28(3): 438-443. 
Oliveira, M.L.J.; Vidal-Torrado, P.; Otero, X.L.; Ferreira, J.R. 2007. Mercúrio total em solos de manguezais da Baixada Santista e Ilha do Cardoso, Estado de São Paulo. Química Nova, 30(3): 519-524.

Paraquetti, H.H.M.; Aires, G.A.; Almeida, M.D.; Molisani, M.M.; Lacerda, L.D. 2004. Mercury distribution, speciation and flux in the Sepetiba Bay tributaries, SE Brazil. Water Research, 38: 1439-1448.

Pereira, S.B. 2000. Aspectos geoquimicos do material particulado da pluma estuarina do rio Amazonas: fatores que controlam as interaçôes com as águas oceânicas. Marter's Thesis, Universidade Federal do Pará, Belém, PA. 130p.

Pfeiffer, W.C.; Lacerda, L.D.; Souza, C.M.M.; Silveira, E.; Bastos, W.R. 1989. Mercury concentrations in inland waters of gold mining areas in Rondônia, Brazil. The Science of the Total Environment, (87/88): 233-240.

Pomerancblum, M.; Costa, M.P.A. 1972. Sedimentologia da Plataforma Norte Brasileira. Anais do XXVI Congresso Brasileiro de Geologia. Belém, Pará, p. 157-178.

Reboreda, R.; Caçador, I.; Pedro, S.; Almeida, P.R. 2008. Mobility of metal in salt marsh sediment colonised by Spartina marítima (Tagus estuary, Portugal). Hydrobiologia, 606: 129-137.

Rocha, J.C.; Sargentini, E.J.; Zara, L.F.; Rosa, A.H.; Santos, A.; Burbad, P. 2003. Reduction of mercury(II) by tropical river humic substances (Rio Negro) - Part II. Influence of structural features. Talanta, 61: 699-707.

Santos, E.C. de O.; de Jesus, I.M.; Brabo, E. da S.; Fayal, K.F.; Sá Filho, G.C.; Lima, M. de O.; Miranda, A.M.M.; Mascarenhas, A.S.; de Sá, L.L.C.; da Silva, A.P.; Câmara, V. de M. 2003. Exposição ao mercúrio e ao arsênio em Estados da Amazônia: síntese dos estudos do Instituto Evandro Chagas/FUNASA. Revista Brasileira de Epidemiologia, 6(2): 171-185.
Siqueira, G.W. 2003. Estudos dos teores de metais e outros elementos em sedimentos superficiais do sistema estuarino de Santos (Baixada Santista - São Paulo) e da plataforma continental do Amazonas (margem continental Norte). Doctoral Thesis, Instituto Oceanográfico, Universidade de São Paulo, São Paulo. 327p.

Siqueira, G.W.; Braga, E.S. 2000. Avaliação da dinâmica e da biodisponibilidade de $\mathrm{Zn}, \mathrm{Ni}$, Co e Pb para a biota a partir de sedimentos da Plataforma Continental do Amapá, nordeste da Amazônia, p. 241-265. In: Espíndolas, E.L.G.; Paschoal, C.M.B.; Rocha. O.; Bohrer, M.B.C.; Oliveira Neto, A.L. (Orgs.). Ecotoxicologia Perspectivas para o Século XXI.

Siqueira, G.W.; Lima, W.N.; Mendes, A.S.; Aprile, F.M.; Braga, E.S.; Lima, E.A.R. 2005. Estudo geoquímico da ocorrência de metais selecionados em sedimentos de fundo da Plataforma Continental do Rio Amazonas - Norte do Brasil, p. 237-245. In. Souza, V.S.; Horbe, A.M.C (Orgs.). Contribuiçôes à Geologia da Amazônia. UFAM, Manaus, AM. v.4.

U.S. Environmental Protection Agency (USEPA). 2003. Revised National Recommended Water Quality Criteria for the Protection of Human Health. Washington, DC, USEPA.

Recebido em 13/02/2011

Aceito em 07/07/2011 
